\title{
PHYSICAL ANALYSIS AND SCALING OF A JET AND VORTEX ACTUATOR
}

\author{
Jason T. Lachowicz \\ Aerospace Engineering Department \\ Old Dominion University \\ Norfolk, VA 23529
}

\author{
Chung-Sheng Yaot \\ Flow Modeling \& Control Branch \\ NASA Langley Research Center \\ Hampton, VA 23681
}

\author{
Ronald D. Joslint \\ Flow Modeling \& Control Branch \\ NASA Langley Research Center \\ Hampton, VA 23681
}

\begin{abstract}
Our previous studies have shown that the Jet and Vortex Actuator generates free-jet, wall-jet, and nearwall vortex flow fields. That is, the actuator can be operated in different modes by simply varying the driving frequency and/or amplitude. For this study, variations are made in the actuator plate and wide-slot widths and sine/asymmetrical actuator plate input forcing (drivers) to further study the actuator induced flow fields. Laser sheet flow visualization, particleimage velocimetry, and laser velocimetry are used to measure and characterize the actuator induced flow fields. Laser velocimetry measurements indicate that the vortex strength increases with the driver repetition rate for a fixed actuator geometry (wide slot and plate width). For a given driver repetition rate, the vortex strength increases as the plate width decreases provided the wide-slot to plate-width ratio is fixed. Using an asymmetric plate driver, a stronger vortex is generated for the same actuator geometry and a given driver repetition rate. The nondimensional scaling provides the approximate ranges for operating the actuator in the free jet, wall jet, or vortex flow regimes. Finally, phase-locked velocity measurements from particle image velocimetry indicate that the vortex structure is stationary, confirming previous computations. Both the computations and the particle image velocimetry measurements (expectantly) show unsteadiness near the wide-slot opening, which is indicative of mass ejection from the actuator.
\end{abstract}

\section{NOMENCLATURE}

$a_{d} \quad$ Actuator plate displacement from maximum position out of cavity to maximum position into cavity (mm)

$b \quad$ Actuator plate width ( $\mathrm{mm}$ )

$d_{y} \quad$ Core vortex size $(\mathrm{mm})$

$d T \quad$ Downward period of actuator plate (s)

${ }^{*}$ Research Assistant Professor

†Senior Research Scientist $\left[d T=1 /\left(2 * f_{\text {rep }}\right)\right.$ for sine wave plate driver]

$1 / d T$ Actuator plate pump rate (1/s)

$f_{\text {rep }} \quad$ Actuator plate driver pulse repetition rate $(\mathrm{Hz})$

$g_{w} \quad$ Ratio of wide-slot width to (actuator) platewidth $=w_{w} / b$

Re Reynolds number $=\frac{\pi a_{d} b}{2 d T v}$

$S_{a} \quad$ Scaled amplitude $=\pi \mathrm{a}_{\mathrm{d}} / \mathrm{b}$

$S t \quad$ Stokes number at wide slot $=\left(2 \pi f_{\text {rep }} w_{w}^{2} / v\right)$

$U^{w} \quad$ Velocity component measured in the $x$ - direction (horizontal) $(\mathrm{m} / \mathrm{s})$

$U_{\mathcal{O}} \quad\left(U_{\max }+\left|U_{\min }\right|\right) / 2 \quad(\mathrm{~m} / \mathrm{s})$

$w_{n} \quad$ Narrow-slot width (mm)

$w_{w} \quad$ Wide-slot width $(\mathrm{mm})$

$y$ Distance perpendicular to actuator plate (mm)

$x$ Distance along width of actuator plate measured from edge of wide slot

$v \quad$ Kinematic viscosity $\left(\mathrm{mm}^{2} / \mathrm{s}\right)$

\section{INTRODUCTION}

A primary goal of active flow control is to develop efficient actuators and sensors that can be integrated with flight control systems to enhance aircraft performance at a reduced cost. Most of the research has focused on surface mounted actuators that either reduce drag, enhance lift, or produce a controlled force about some axis of the aircraft. Active control devices (Wiltse and Glezer, 1993; Amitay and et al., 1998; Compton and Johnston, 1992; Magill and McManus, 1998) produce no parasitic drag and can be effectively used for multiple flight conditions unlike passive devices (Bragg and Gregorek, 1987; Lin et al., 1994) which are typically optimized for a single flight condition. Zero-net-mass flux actuators (Wiltse and Glezer, 1993; Amitay et al., 1998) are especially attractive since they require no internal ducts or 
pumps. This has the potential to significantly reduce the mass of a vehicle and decrease the aircraft signature. The Jet and Vortex Actuator or JaVA (Lachowicz et al., 1998, 1999) is one type of zero-netmass flux actuator. Compared with other zero-netmass flux actuators such as the synthetic jet (Amitay et al., 1998), the mechanisms of generating vorticity are different. While the synthetic jet generates two symmetric vortices of opposite direction, the JaVA generates a single stationary vortex.

The JaVA consists of a cavity and rigid plate that serves as the actuation surface. The plate is oscillated vertically as shown in Figure 1 such that the plate motion is displaced uniformly across it's length and width. The plate acts like a piston pumping air out of the cavity on the down-stroke and sucking air into the cavity on the upstroke. The plate is placed asymmetrically over the cavity opening, forming narrow and wide slots when viewed from the top as shown in Figure 1. Originally, Jacobson and Reynolds (1995, 1998) used a flow control actuator similar to the JaVA, but their actuator was cantilevered along the width of the plate, producing a variable displacement across the length of the plate. In their experiments, a periodic jet was generated from the narrow slot of the actuator. In the experiments of Saddoughi et al (1998), however, a periodic jet was generated from the wide slot. To resolve this discrepancy, Koumoutsakos (1995) conducted numerical simulations of the actuator assuming it provided a uniform displacement across the length of the plate (2D). The $2 \mathrm{D}$ computational results showed, qualitatively, that a jet emerges from either slot depending on the relative amplitude and frequency of actuation.

Lachowicz et al. (1998, 1999) characterized, in detail, the JaVA flow. In still air, it was found that several flow fields were produced by the JaVA (wall jet, free jet, and vortex flow). The flow fields were found to depend on actuator Reynolds number, scaled amplitude, and $g_{w}$ when using sine wave forcing to drive the actuator plate. The optimum actuator efficiency occurred at a Stokes number of approximately 7.9 for the vortex flow over the range of parameters studied. Since the JaVA produces several flow regimes, it is potentially suited for more than one application. However, this research focuses primarily on the vortex flow where the JaVA functions as an active vortex generator. Recent computational results (Joslin et al., 1998) were shown to be in good agreement with the experimental results for the vortex induced flow case.

In this study, the JaVA is examined using laser velocimetry, particle image velocimetry, and laser sheet flow visualization. The main objectives of the research are to study the JaVA induced vortex flow field using asymmetric forcing to drive the actuator plate, and to determine the nondimensional scaling of the stand-alone JaVA.

\section{APPROACH}

\section{Flow Control Actuator}

The experimental configuration of the JaVA is discussed in detail by Lachowicz et al. (1999). All previously published results for the JaVA used a sine wave to drive the actuator plate. Similar to the present study, the plate was driven off resonance over a range of frequencies using a mechanical oscillator. For the present study, it is postulated that some form of asymmetric forcing may lead to a stronger induced vortex because the vortex generation mechanism relies primarily on the downward stroke of the actuator plate motion. For asymmetric forcing, the duration and form of the positive and negative strokes of the actuator plate differ. Whereas, the sine wave forcing has equal duration and amplitude on both the positive and negative strokes. Various asymmetric forcing waves were initially studied but only results using a pulsed wave train to drive the actuator plate are presented in this paper.

\section{Measurements}

Flow visualization, actuator displacement measurements, and frequency measurements were conducted simultaneously to document the actuator flow field in still-air. The laser-sheet flow visualization system consisted of a 3 Watt Argon-Ion laser and a progressive scan video camera. The images were recorded in analog form on tape and also in digital form on computer. The actuator displacements were measured using a fiber-optic displacement sensor. The displacement measurements have an uncertainty of $\pm 4 \%$ of the measured value. The nondimensional scaling was determined from these measurements.

A 2-component LV system was used for point velocity measurements of the actuator flow field. Particles on the order of 1 micron were used for seeding. The LV probe was scanned in several crossplanes to provide mean velocity profiles perpendicular to the actuator plate surface. The LV velocity uncertainty is $\pm 0.06 \mathrm{~m} / \mathrm{s}$, and the data were not corrected for velocity biasing. The global vorticity was estimated from these measurements.

A digital PIV system was used to measure the instantaneous flow field in the $\mathrm{x}-\mathrm{y}$ plane perpendicular to the actuator plate surface. The flow field measurement was phase locked to eight equally spaced phases (45 degree intervals) of the actuator plate motion. Particle images are recorded using a digital camera $(1 \mathrm{Kx} 1 \mathrm{~K}$ pixels) with a $105 \mathrm{~mm}$ lens at $1 / 3$ image reduction ratio. Measurement volume spatial resolution is less than $1 \mathrm{~mm}$ at the test plane and 32 pixels at the image plane. Ten samples of PIV images were taken at each phase. Ensemble averages 
were computed to estimate both phase-averaged and over-all averaged velocity fields.

\section{Computations}

The computational approach (Joslin et al., 1998) uses fourth-order finite central differences in both $\mathrm{x}$ and $y$ directions and a low-storage three-stage Runge-Kutta method for time-advancement. Parameter studies were conducted to ensure spatial and temporal resolution of the induced flow fields.

\section{RESULTS}

In still-air, the JaVA generates several flow regimes as illustrated in Figure 2. In this study, the focus is primarily on the vortex flow regime characterized by a stationary vortex from the wide slot. Figure 3 shows a typical time-averaged visualization for the vortex-induced flow field; the arrow denotes the direction of the vortex. For this case, the vortex spans approximately 50 to $60 \%$ of the plate width as observed from the flow visualization. But, the core vortex size, estimated from the velocity profiles, is approximately 30 to $35 \%$ of the plate width.

For $f_{\text {rep }}=50 \mathrm{~Hz}$, the actuator plate motion using (pulsed wave train) asymmetric forcing is shown in Figure 5. The actuator plate is pumped over a short duration but a large stroke into the cavity, with a long duration but a small stroke out of the cavity. For this forcing, the JaVA is operated in the vortex flow regime.

The vortex flow velocity profiles are compared using asymmetric and sine-wave forcing in Figure 5 at $g_{w}=0.098$; the computational results are represented by the solid lines. The core vortex size, $d_{y}$, is estimated as the distance between the velocity maxima and is illustrated in the figure. Assuming a symmetric vortex, the maximum mean vorticity is estimated as 4 $U_{\delta} / d_{y}=2 *\left(U_{\max }+\left|U_{\min }\right|\right) / d_{y}$. Comparing the sine-wave driver to the asymmetric driver at $f_{\text {rep }}=130 \mathrm{~Hz}$, the core vortex size is smaller for the asymmetric driver yet the velocity maxima are approximately the same. This suggests that asymmetric forcing provides a larger vorticity level than sine forcing at a fixed $g_{w}$ and repetition rate.

The actuator vorticity performance over a range of actuator and plate widths using both sine forcing and asymmetric forcing is presented in Figure 6. As described previously, the maximum mean vorticity is estimated as $4 U_{o} / d_{y}$ and is represented along the yaxis. The asymmetric driver is represented by the $w_{w}=0.96 \mathrm{~mm}(b=9.85 \mathrm{~mm})$ case and the maximum repetition rate for this case is approximately $130 \mathrm{~Hz}$; all other cases use the sine-wave driver. Generally, the vorticity increases with repetition rate for both drivers at a fixed $w_{w}$ and $b$. Assuming an extrapolation of the data is valid, the present data indicates that large potential gains in vorticity can be obtained at large repetition rates. The different drivers are compared at $w_{w} \approx 0.96 \mathrm{~mm}$ for approximately the same plate width (open triangle and $\mathrm{x}$-symbols). For example, at $f_{\text {rep }}=130 \mathrm{~Hz}$, the vorticity is approximately $75 \%$ larger for the asymmetric driver. Overall, for the same plate size and a given repetition rate, the vortex strength increases for the asymmetric driver relative to the sinedriver for $w_{w} \approx 0.96 \mathrm{~mm}$.

Figure 6 also shows the dependency of the vorticity as a function of the plate width size. For $f_{\text {rep }}$ $<125 \mathrm{~Hz}$ (limit of data available for $b=5.56 \mathrm{~mm}$ ), the vorticity is much larger for the smallest plate width relative to the larger plate widths. For example, for $g_{w}=w_{w} / b \approx 0.17$ (plus and square symbols), the vorticity for the $b=5.56 \mathrm{~mm}$ case is approximately $115 \%$ larger than the $b=9.65 \mathrm{~mm}$ case at a given piston pump rate. However, the velocity data indicate that the core vortex size decreases by about $15 \%$ with $b$ for these cases. So, a large increase in vortex strength occurs with only a marginal decrease in vortex size. The same trends occur for $g_{w} \approx 0.10$ at $b=5.56$ (filled circle) and $9.65 \mathrm{~mm}$ (open triangle). Overall, the vortex strength increases as the plate width decreases for a fixed $g_{w}$ and repetition rate. The trend-lines in Figure 6 are only approximate as figure 2 shows the vortices exist over a region.

The non-dimensional parameters governing the JaVA were derived in Lachowicz et al. (1999). The analysis assumed 2D incompressible flow, a fixed narrow slot width, and a characteristic velocity equal to the actuator plate peak velocity. Under these assumptions, the non-dimensional parameters governing the JaVA are $\operatorname{Re}=\frac{\pi a_{d} b}{2 d T v}, S_{a}=\pi a_{d} / \mathrm{b}$, and $g_{w}=w_{w} b$ for either the sine wave or asymmetric plate driver. (Note that $R e=\pi f a_{d} b / v$ for a sine wave driver). Figure 2 showed the different flow regimes plotted in terms $R e$ and $S_{a}$ at a fixed $g_{w}$ and the general relationship between these governing parameters is shown in Figure 7 for three flow regimes: wall jet, free jet, and vortex flow. (Note that this data was conducted over an actuator plate width range from 5.56 $\mathrm{mm}$ to $18.8 \mathrm{~mm}$ and a wide slot range from $0.32 \mathrm{~mm}$ to $2.54 \mathrm{~mm}$ ). Although overlap exists between each region, each flow field has a region that is unique to that particular flow field. Operation of the actuator in this unique region would produce the desired flow of interest. For example, at $\operatorname{Re} g_{w}=25, \sqrt{\frac{R e}{S_{a}}} g_{w}^{2}=0.5$ and 1 for the vortex flow and wall jet, respectively. For $\operatorname{Re} g_{w}=4$, the free jet exists at $\sqrt{\frac{R e}{S_{a}}} g_{w}^{2}=0.07$. The nondimensional range for asymmetric forcing is within the range for sine forcing for the vortex flow. This suggests that, although the vortex strength 
depends on the forcing frequency (Fig. 6), the scaling is not dependent on the type of forcing.

Figures 8 to 10 show the phase-averaged velocity fields for the actuator operating in the vortex mode. Each figure represents the average of 10 samples at a particular phase of the actuator plate oscillation. A vortex structure is measured above the piston for each phase suggesting that the structure is stationary; note that similar results are observed for the additional five phases not presented. This is consistent with the averaged flow visualization pattern observed earlier, Figure 3, and the computations (Joslin et al., 1998). In addition, an unsteady angled jet flow is pumped from the wide gap indicative of mass ejection from the actuator. Figure 11 shows the averaged flow field over eight phases. A single cell vortex structure is clearly observed, verifying that the vortex is dominated by a stationary mode.

\section{CONCLUDING REMARKS}

A parameter study of the Jet and Vortex Actuator has been conducted for the vortex flow field. The actuator plate forcing and size were changed to increase the strength of the vortex generated by the stand-alone actuator. For the range of parameters surveyed, the measurements indicate that the vortex strength increases with driver repetition rate for a fixed wide slot and plate width. For a given driver repetition rate, the vortex strength increases as the plate width decreases provided the wide-slot to plate-width ratio is fixed. Using an asymmetric plate driver, a stronger vortex is generated for the same actuator geometry (i.e. same slot widths and actuator plate width) and a given driver repetition rate. The nondimensional scaling provides the approximate ranges for operating the actuator in the free jet, wall jet, or vortex regimes. The phase-locked velocity measurements from particle image velocimetry indicate that the vortex structure is stationary, confirming previous computational results.

\section{ACKNOWLEDGEMENTS}

This work was performed while the first author held a National Research Council Post-Doctoral Fellowship at NASA-Langley Research. This work was supported by the Aircraft Morphing element of the NASA Airframe Systems Program Office and by the AFOSR.

\section{REFERENCES}

Amitay, M., Smith, B.L., and Glezer, A., "Aerodynamic Flow Control Using Synthetic Jet Technology," AIAA 98-0208, Jan. 1998.
Bragg, M.B. and Gregorek, G.M., "Experimental Study of Airfoil Performance with Vortex Generators," J. Aircraft, Vol. 24, No. 5, May 1987, pp. 305-309.

Compton, D.A. and Johnston, J.P., "Streamwise Vortex Production by Pitched and Skewed Jets in a Turbulent Boundary Layer," AIAA Journal, Vol. 30, No. 3, March 1992, pp. 640-647.

Jacobson, S.A. and Reynolds, W.C., "An Experimental Investigation Towards the Active Control of Turbulent Boundary Layers," AFSOR Report Number TF-64, March 1995.

Jacobson, S.A. and Reynolds, W.C., "Active Control of Streamwise Vortices and Streaks in Boundary Layers," J. Fluid Mech., Vol. 360, April 1998, pp. 179-212.

Joslin, R.D., Lachowicz, J.T., and Yao, C.-S., "DNS of Flow Induced by a Multi-Flow Actuator," ASME Paper FEDSM98-5302, June 1998.

Koumoutsakos, P., "Simulations of Vortex Generators," Center for Turbulence Research, Annual Research Briefs, 1995, pp. 233-240.

Lachowicz, J.T., Yao, C.-S., and Wlezien, R.W., "Scaling of an Oscillatory Flow Control Actuator," AIAA 98-0330, Jan. 1998.

Lachowicz, J.T., Yao, C., and Wlezien, R.W., "Flow Field Chacterization of a Jet and Vortex Actuator," Experiments in Fluids, (accepted) 1999.

Lin, J.C., Robinson, S.K., McGhee, R.J., and Valarezo, W.O., "Separation Control on High-Lift Airfoils via Micro-Vortex Generators," Journal of Aircraft, Vol. 31, No. 6, 1994, pp. 1317-1323.

Magill, J.C. and McManus, K.R., "Control of Dynamic Stall Using Pulsed Vortex Generator Jets," AIAA 98-0675, Jan. 1998.

Saddoughi S.G., Koumoutsakos, P., Bradshaw, P.; and Mansour, N.N., "Investigations of On-Demand Vortex Generators," Center for Turbulence Research Manuscript No. 171, Stanford Univ., Sept. 1998.

Wiltse, J.M. and Glezer, A., "Manipulation of Free Shear Flows Using Piezoelectric Actuators," J. Fluid. Mech., Vol. 249, 1993, pp. 261-285. 
Top View

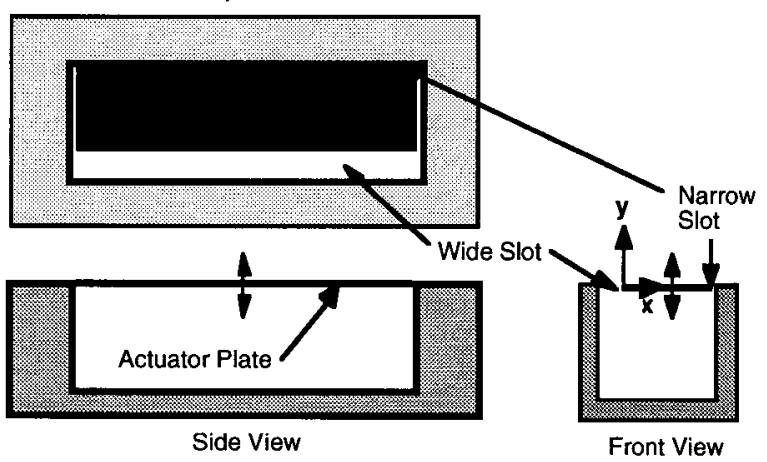

Figure 1. Schematic diagram of JaVA

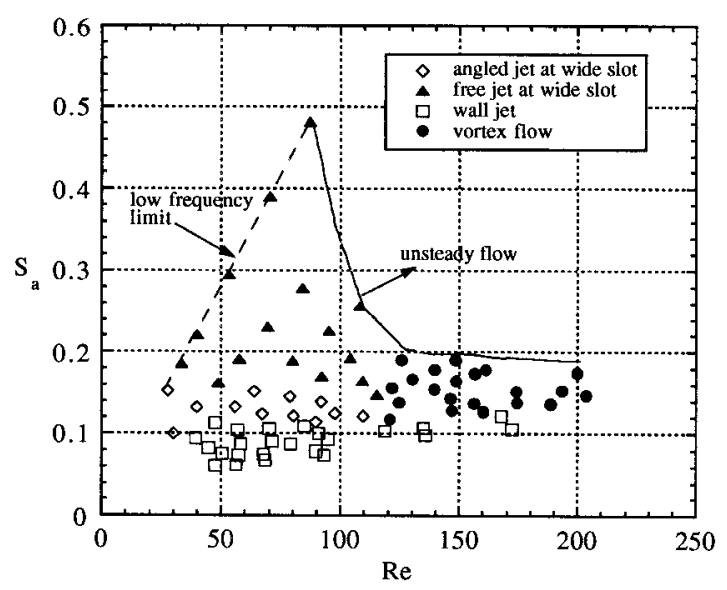

Figure 2. Flow regimes with $g_{w}=0.098$ (Lachowicz et al, 1999)

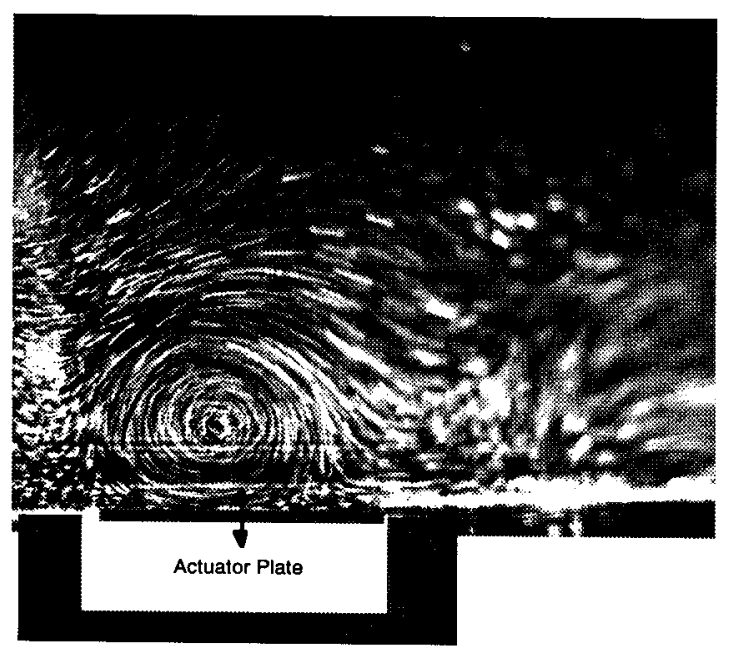

Figure 3. Typical flow visualization of vortex flow

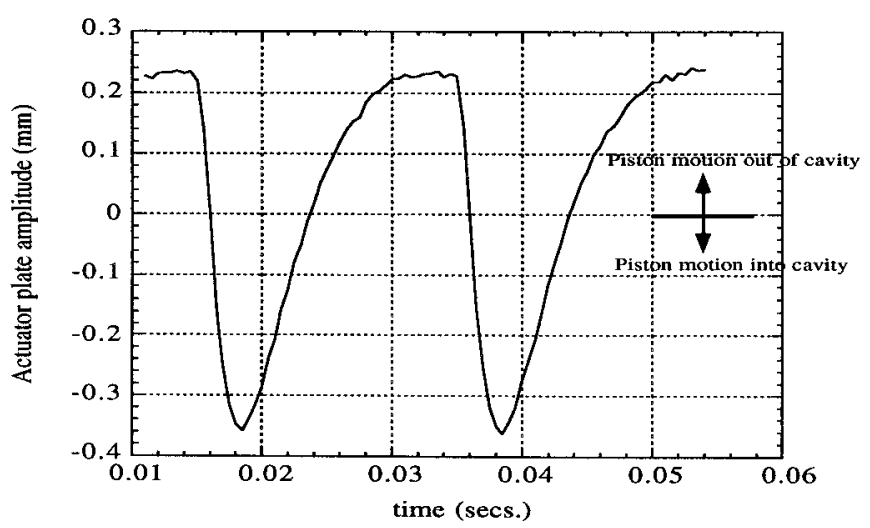

Figure 4: Actuator plate motion using asymmetric forcing to drive JaVA in vortex flow regime, $t_{\text {rep }}=50 \mathrm{~Hz}$

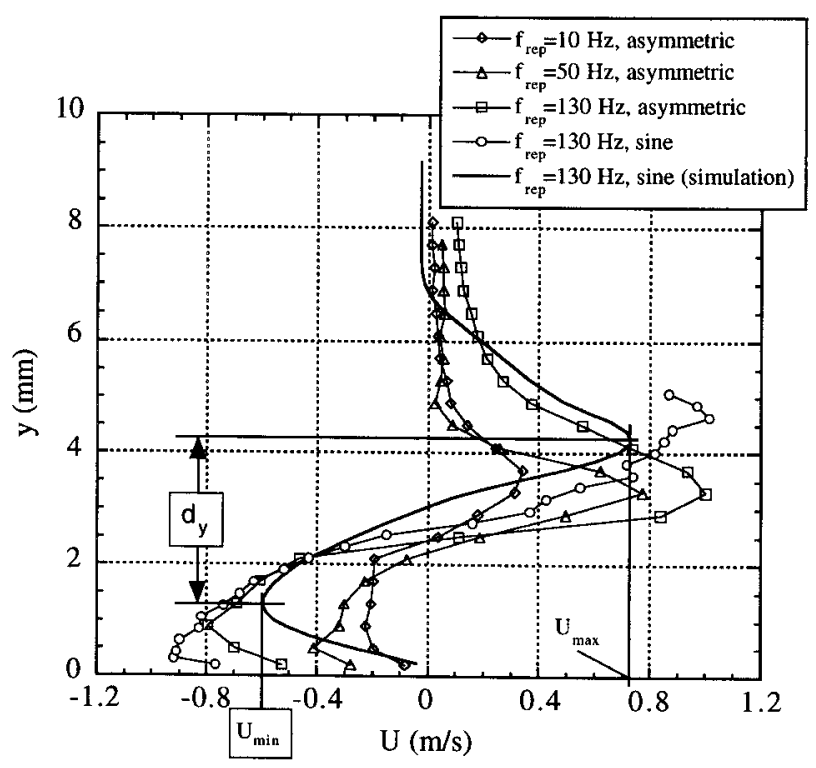

Figure 5: Velocity profiles at $x / b=0.10$, $g_{w}=0.098$ 


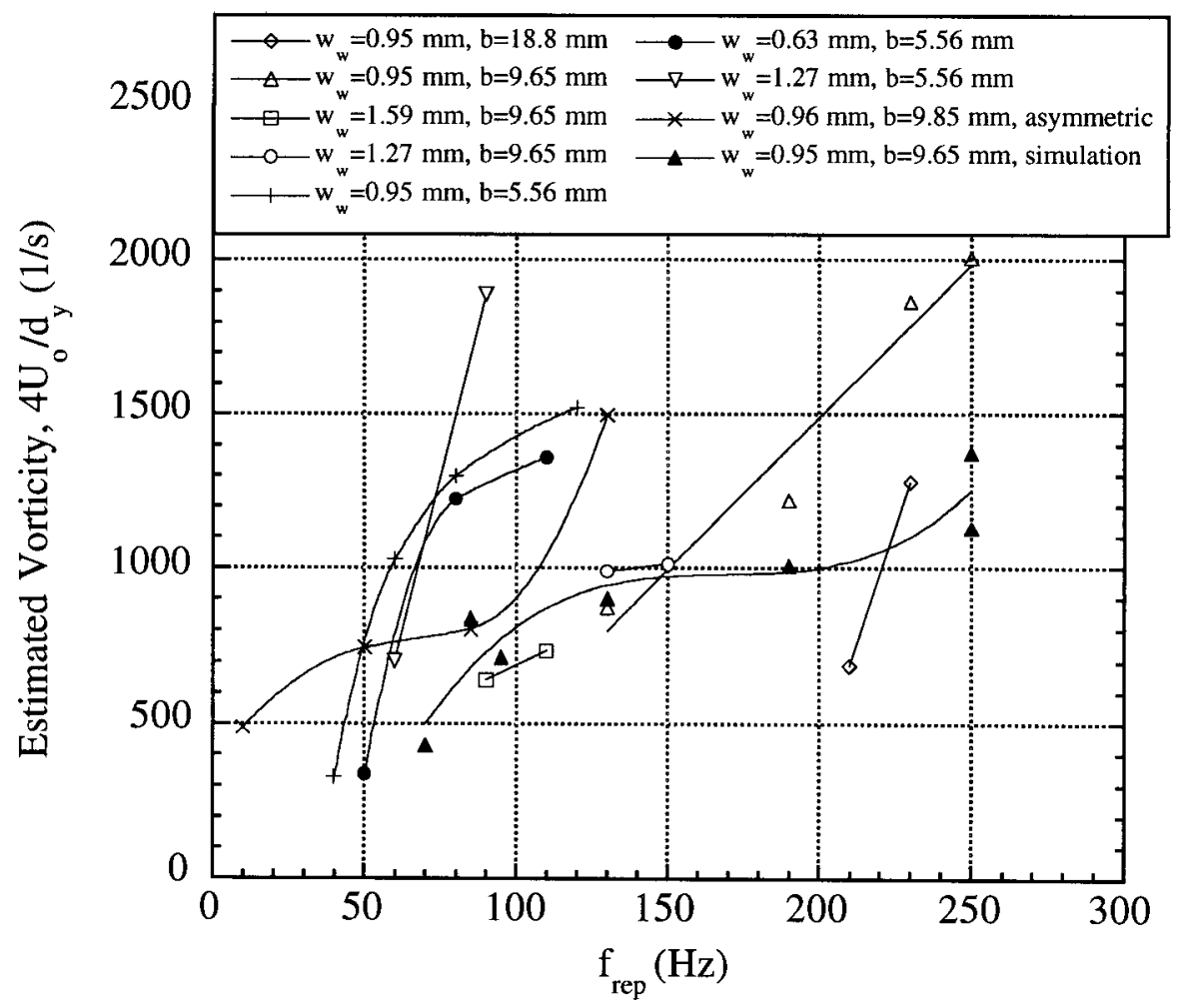

Figure 6: Estimated vorticity as function of repetition rate

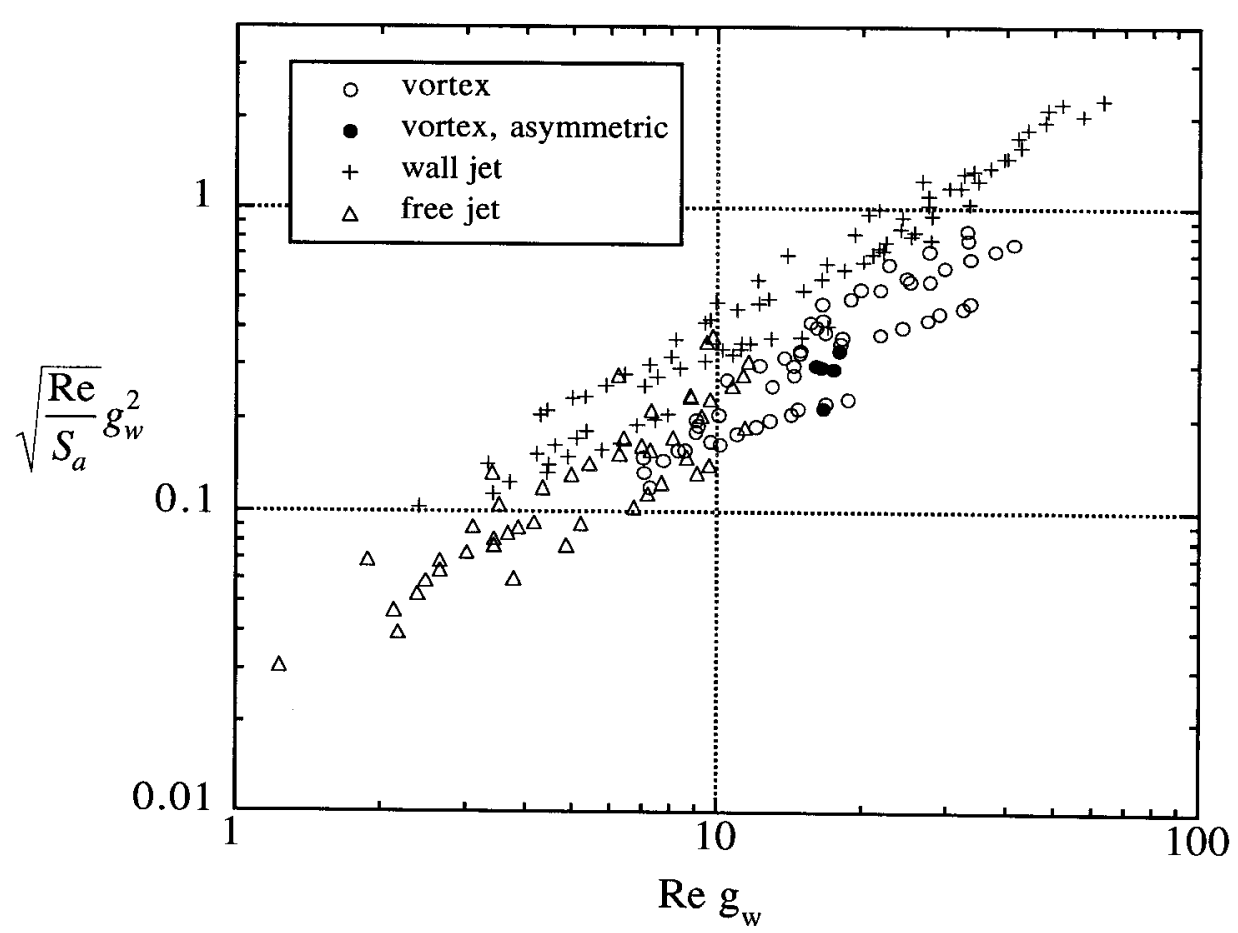

Figure 7. Non-dimensional scaling for JaVA 


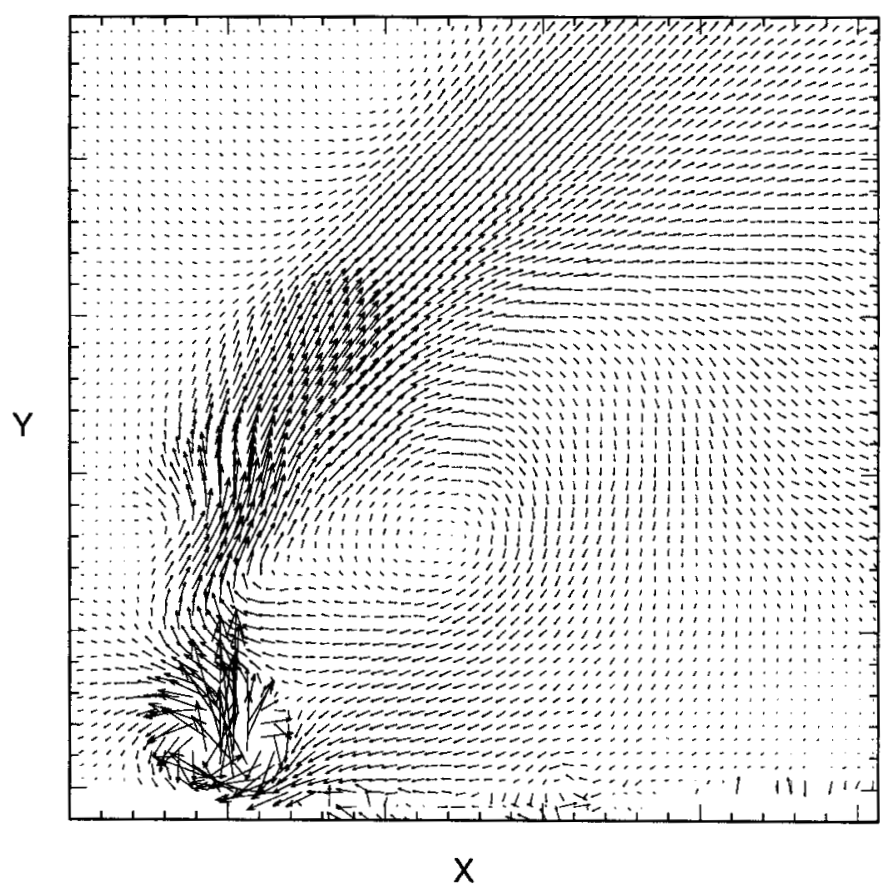

Figure 8. Phase-averaged velocity field: Phase 1 (plate into cavity)

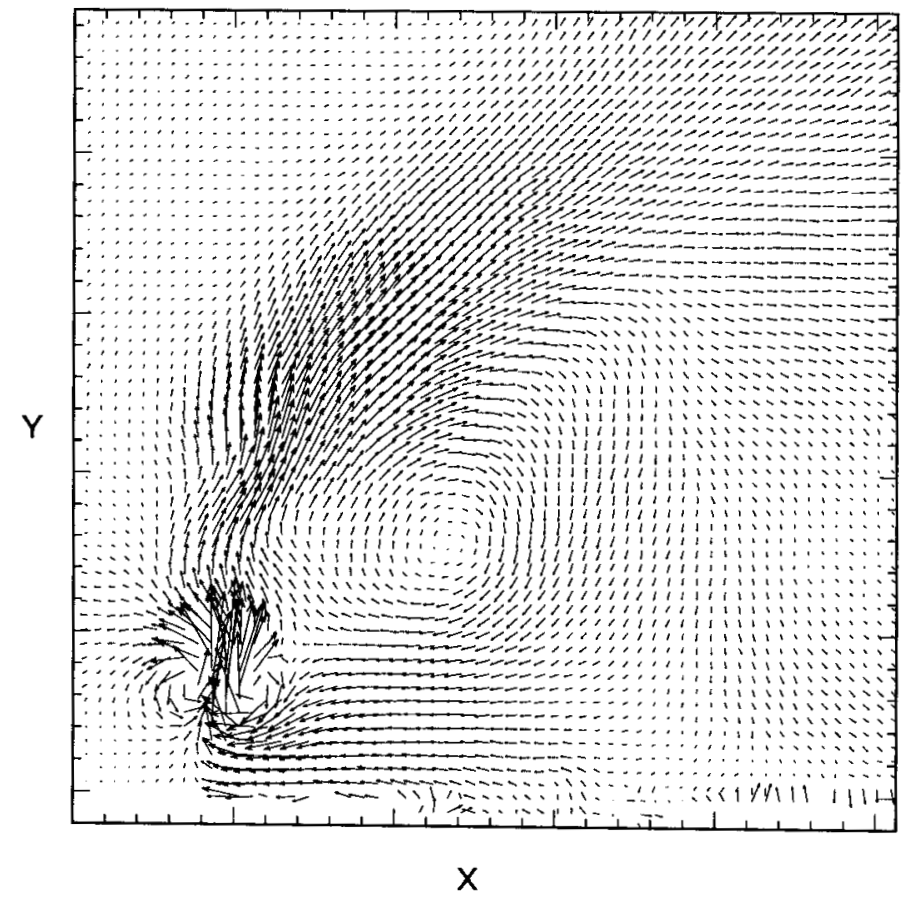

Figure 9. Phase-averaged velocity field: Phase 2 (plate flush with top of cavity) 


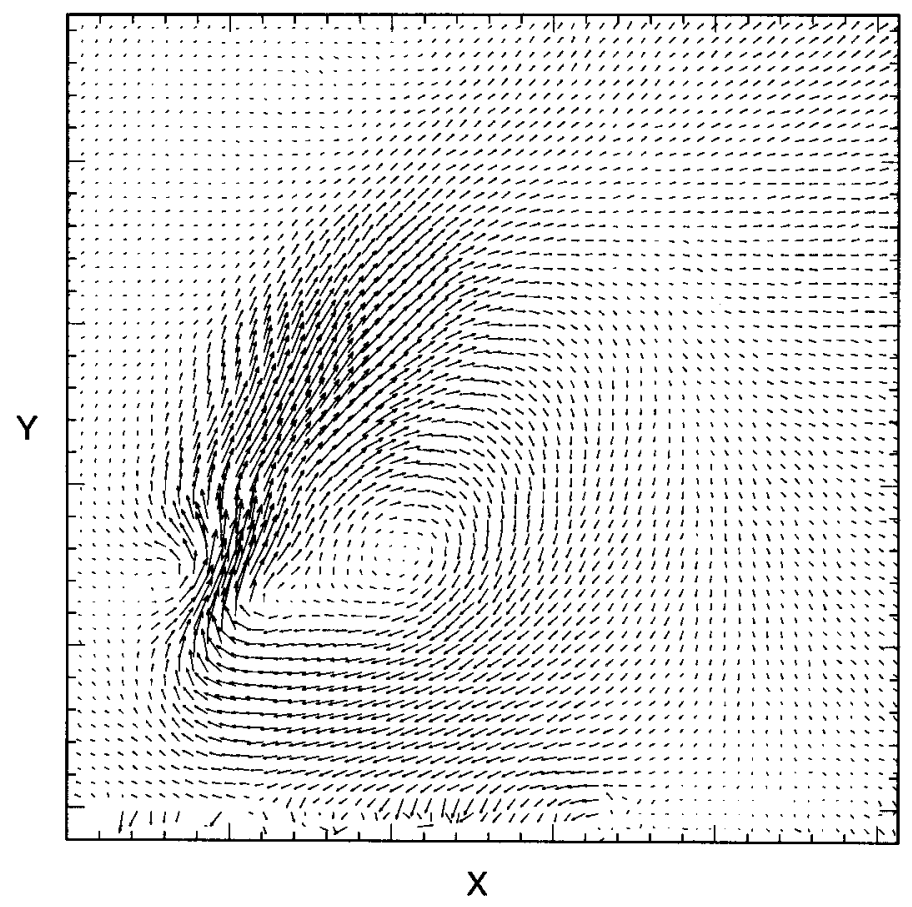

Figure 10. Phase-averaged velocity field: Phase 3 (plate out of cavity)

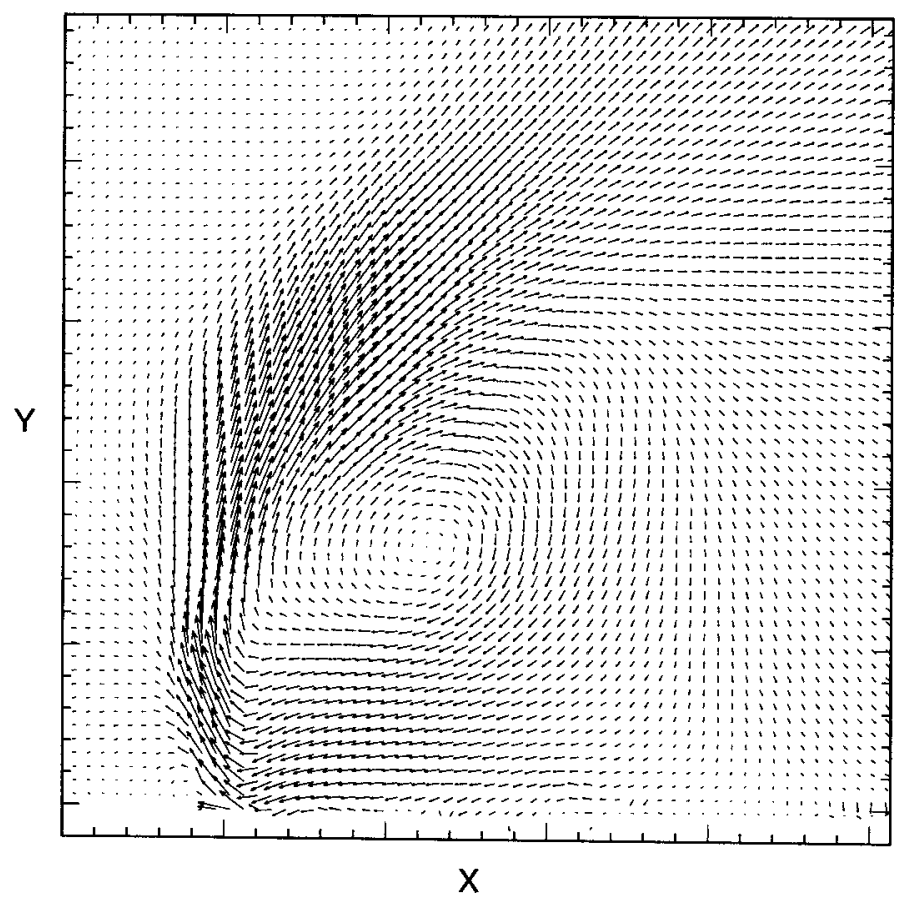

Figure 11. Mean velocity field 